RESEARCH HIGHLIGHTS

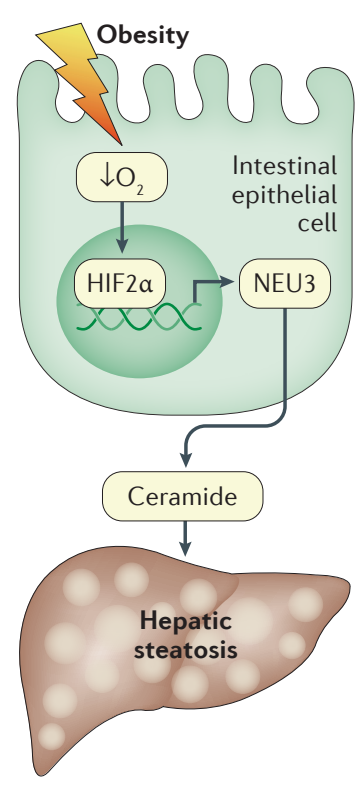

Intestinal HIF2 $\alpha$ is induced in obese humans and mice, and the intestinal HIF2 $\alpha-N E U 3$ pathway controls serum ceramide levels, which in turn affect NAFLD development.

NAFLD

\title{
HIF2a: a new therapeutic target for NAFLD
}

A new study has shown that intestinal hypoxia-inducible factor (HIF) $2 \alpha$ signalling is activated in obesity, promotes hepatic steatosis in mice and might be a promising therapeutic target for NAFLD in humans.

Obesity is a well-recognized risk factor for NAFLD, the most common liver disorder worldwide. However, no safe and effective drug therapy is currently available for NAFLD. Previous work demonstrated that intestine-derived ceramide can trigger the development of hepatic steatosis and that adipocyte-specific inhibition of HIF improves hepatic steatosis. Now, this study provides a link between intestinal HIF, ceramide metabolism and NAFLD.

Using distal ileum biopsy samples from obese and non-obese individuals, the investigators first showed that intestinal HIF $2 \alpha$ signalling was positively correlated with BMI and hepatic toxicity.
"A genetic, pharmacological and high-throughput global lipidomics approach was then used to dissect how intestinal HIF2 $\alpha$ regulates hepatic steatosis," explains author Frank Gonzalez.

In mice fed a high-fat diet (HFD) and with intestine-specific disruption of Hif2a, levels of hepatic steatosis and obesity were markedly lower than in HFD-fed control mice. Lipidomics was used to analyse the intestinal metabolites of these mice. "Ceramide was the top determinant among lipids derived from the wild-type and intestine-specific Hif $2 \alpha$ knockout mice," reports Gonzalez.

Further genetic mouse models established that Neu3 was the most robustly induced gene involved in ceramide synthesis following HIF2 $\alpha$ activation. Expression of NEU3 was also increased in human ileum biopsy samples from obese individuals. Together, these results suggest the intestinal HIF2 $\alpha$-NEU3-ceramide axis promotes NAFLD. To test this hypothesis, HFD-fed mice with hepatic steatosis were treated with PT2385, a HIF2 $\alpha$-specific inhibitor. This approach substantially reversed hepatic steatosis in this mouse model.

"Our study identified an essential role for intestinal HIF2 $\alpha$ in controlling fatty liver ... and provides a potential therapeutic approach to treat NAFLD," concludes author Changtao Jiang. "PT2385 is already used in clinical trials for renal cell carcinoma. It will be of great interest to determine the role of intestinal HIF $2 \alpha$ and PT2385 in the treatment and progression of cirrhosis and hepatocellular carcinoma."

Iain Dickson

ORIGINAL ARTICLE Xie, C. et al. Activation of hypoxia-inducible factor $2 a$ during obesity contributes to hepatic steatosis. Nat. Med. http://dx.doi.org/10.1038/nm.4412 (2017) 